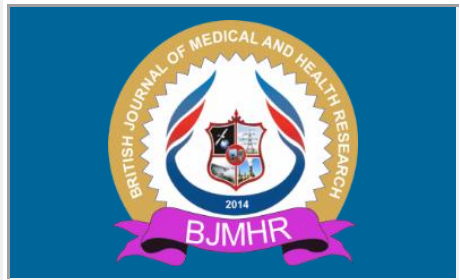

\title{
BJMHR
}

British Journal of Medical and Health Research Journal home page: www.bjmhr.com

\section{Cognitive Impairment and Memory Loss in Diabetes Mellitus:} A Systematic Review

\author{
Madeeha Malik, Sidra Bi, Azhar Hussain \\ Professor/Director, Hamdard Institute of Pharmaceutical Sciences, Hamdard University \\ Islamabad Pakistan.
}

\begin{abstract}
Uncontrolled diabetes for prolong period results in cognitive decline and memory loss. Decline in effectiveness of processing resources, thinking ability and to recall objects is thought to be a result of mismanagement of diabetes. Early identification of cognitive impairment (CI) and memory loss allows for timely prevention and better prognosis for patients and their caretakers. The aim of this study is to summarize research findings from developed and developing countries as well as from Pakistan regarding cognitive impairment and memory loss in diabetes mellitus. A total of 58 studies were reviewed regarding cognitive impairment and memory loss among diabetics. The review concluded that for successful management of diabetes, it is important to identify the degree of cognitive impairment and memory loss in patients. Regular screening for cognitive impairment among diabetes patients must be conducted in order to provide optimal patient care.
\end{abstract}

Keywords : Cognition, memory loss, diabetes mellitus, Pakistan

*Corresponding Author Email: madeehamalik15@gmail.com

Received 07 October 2018, Accepted 17 October 2018

Please cite this article as: Malik M et al., Cognitive Impairment and Memory Loss in Diabetes Mellitus: A Systematic Review. British Journal of Medical and Health Research 2018. 


\section{INTRODUCTION}

Diabetes mellitus is a common metabolic disorder with high blood sugar levels for prolong time period. Chronic complications such as cardiovascular disease, nephropathy, retinopathy, and peripheral neuropathy could result if diabetes is left untreated (1). Cognition refers to the mental capabilities or thinking skills that allow a person to perceive, acquire, understand and respond to information from their environment (2). The incidence of mild cognition impairment has been estimated to be 5-20\% among people older than 65 years. However, people with diabetes are more prone towards this issue. This progressive loss of cognitive performance contributes to intellectual, behavioural, and functional declines and an inability to learn. Many types of chronic illness related to cognitive impairment, including depression, stroke, cardiovascular disease, and hyperglycaemia have been reported among elderly with diabetes (3).Uncontrolled diabetes for prolong period results in cognitive decline and memory loss. Decline in effectiveness of processing resources, thinking ability and to recall objects is thought to be a result of mismanagement of diabetes (1).

Early identification of cognitive impairment (CI) and memory loss allows for timely prevention and better prognosis for patients and their caretakers .However, effective approach to identify cognitively impaired patients from community for interventions is not enough, due to lack of routine cognitive screening. Beside diabetes some other risk factors, such as poor dietary approaches, hypertension, high cholesterol, and smoking, as well as cerebrovascular diseases and changes in neuronal receptors have shown similar connection with cognitive impairment. These risk factors together with age have strong impact on cognitive impairment. Therefore, these factors can help in identifying older adults at risk of CI in primary health care (4).The aim of this paper is to highlight the prevalence of cognitive impairment and memory loss among diabetes patients by reviewing the past and present literature. Furthermore, it will systematically identify and review the impact of cognitive impairment and memory loss among diabetics as well as interventions to improve the impairment worldwide.

\section{MATERIALS AND METHOD}

The electronic databases PubMed, Google Scholar, and Science Direct, were searched for articles published from 2000 to 2017 . The search terms used with each database were diabetes, cognition and memory loss. Full-text papers, as well as abstracts, were retrieved and included in review. A total of 58 studies were retrieved from databases related to diabetes and cognition impairment. The studies were categorized on the basis of their country of publishing into developed countries, developing countries, and Pakistan. 40 studies from developed countries, 17 from developing countries, and 1 study from Pakistan were included in this review (Table 
1). Quantitative cross-sectional surveys, as well as qualitative studies, were also included in this study.

Table 1 Countries and Number of Included Studies

\begin{tabular}{|c|c|c|}
\hline Regions & $\begin{array}{l}\text { Number of studies } \\
\text { included }\end{array}$ & Countries \\
\hline $\begin{array}{l}\text { Developed } \\
\text { countries }\end{array}$ & 40 & $\begin{array}{l}\text { USA, Australia, UK, Japan, Germany, Belgium, } \\
\text { Switzerland, Sweden, Canada, Denmark, Greece, } \\
\text { Spain, Italy, Finland, Ireland, Netherlands }\end{array}$ \\
\hline $\begin{array}{l}\text { Developing } \\
\text { countries }\end{array}$ & 17 & $\begin{array}{l}\text { Turkey, China, South Africa, Ghana, Malaysia, } \\
\text { India, Taiwan, Iran, Qatar, Eritrea, Jordan }\end{array}$ \\
\hline Pakistan & 1 & \\
\hline
\end{tabular}

\section{RESULTS AND DISCUSSION}

\section{Cognitive Impairment and Memory Loss in Diabetes}

Impaired insulin signalling plays an important role in the pathogenesis of both cognitive and diabetic complications. Uncontrolled diabetes due to impaired insulin signalling is an increased risk of memory loss. The early identification of cognitive impairment can help health care providers in management of complications. If diabetes patients are not managed properly, then they are at a higher risk of developing Alzheimer disease, dementia and other irreversible memory loss. While early detection of symptoms in diabetics allow to implement a personalized medicine, which is an essential issue in diabetes care with significant therapeutic inferences (5).Severe hypoglycaemia as an indication of poor control in diabetes patients was reported to be a stimulus of cognitive impairment and memory loss. HbA1c has reported as a dependent variable while insulin as independent factor. Mini mental state examination tool was used for assessment of cognitive performance, score in the insulin treated patients was significantly lower than that in the untreated patients. In most of cases severe hypoglycaemia was reported in insulin treated group, in this way hypoglycaemia and uncontrolled diabetes both can relate to the loss of memory (5).

A review of literature highlights that community dwelling individuals without evidence of severe functional impairment at baseline, diabetes increases the risk of incident disability in only 2 years. Diabetic patients had an increased risk of any new disability and dependence. Diabetes can reduce insulin sensitivity and relative insulin deficiency leading to cognitive decline (6).In diabetes patients, insulin resistance is considered as increased risk factor for cognitive defect. A strong evidence from a study conducted in USA indicated that memory loss may be a slow progressing brain metabolic disease (7).Individuals with Metabolic syndrome and obesity, have a higher risk of developing memory loss leading to Alzheimer's, while Alzheimer's disease (AD) patients often develop hyperglycaemia. Intranasal insulin enhance 
memory and brain functioning however, is only effective in early memory loss and mild cognitive impairment $(8,9)$.

Unfortunately, medication used for treatment of anxiety and depression in elderly population do not essentially improve the symptoms of cognitive decline, that's why recognition of potentially modifiable risk factors and the preventive strategies for cognitive decline are of major importance for health care providers. Young patients with type I diabetes who are reported with relatively frequent episodes of severe hypoglycaemia showed decline in cognitive ability. However, severe hypoglycaemia is relatively common in adults with insulin treated type II diabetes patients and to a lesser extent in individuals treated with sulfonylurea showing a strong association of treatment induced hypoglycaemia with cognitive impairment(10).

\section{Tool for Cognitive Impairment assessment among Diabetes Patients}

Several tools have been validated for assessment of cognitive impairment in low and high income countries as well as in low-literacy settings. Several tools and tests have been inadequately validated, however only MMSE, cognitive abilities screening instrument (CASI) and Euro test have evolved to get reliable results (11).According to another study assessment of Cognitive performance and functional dependency to identifying memory loss in primary care performed by the Mini Mental State Examination (MMSE) and the General Practitioner assessment of Cognition. Authentic results were shown through direct comparisons of primary outcome by comparing accuracy of the two tests assessed within the same population. Additionally, both primary care physicians and psychiatrists recognised the importance (12).

\section{Overview of Cognitive Decline among Diabetes Patients in Developed Countries}

Health Related Quality of Life, Neurological Changes with Cognitive Impairment and Memory Loss in Diabetes Mellitus. A Study conducted in Netherland assessed the association of metabolic disorder with cognitive decline. Cognitive impairment was associated with some domains of health related quality of life and self-management of diabetes, as the cognitive impairment score increased, quality of life and self-management profile increased, as well. Also, a decreasing trend was observed in HbA1c levels in moderate to normal cognitive impairment states (13). Diabetic complications and mortality rate were greater among depressed patients. Micro and macro vascular complications of diabetes can be considered as a cause of depression among patients with uncontrolled diabetes .Such patients were found more prone towards depression and had decline in cognitive ability. Cognitive complications in patients with diabetes increase with depression thus contributing to the increased mortality rate in this population. Beside this small vessel diseases in the brain affect cognitive function in older diabetics (14). An investigational research study conducted in Finland reported that 
mild cognitive impairment is associated with late detection and poor health along with appropriate disease management leading to dependency in older population. Observational study suggested that poor physical health might be involved in the very early stages of cognitive worsening. Individual with non-neurological conditions which are clinically evident can show selective cognitive problems.

\section{Poor Health Control and Cognitive Decline}

Mild cognitive impairment and memory loss is associated with poor health and unhealthy life style. Mechanism to determine relative contribution of physical and brain factors in development of mild cognitive impairment is not clear. The influence of poor physical health on brain functioning and performance is well known in the clinical practice. Chronic conditions such as diabetes, vascular disease and its complications like neuropathy can lead to poor cognitive performance. Clinically serious but stable and mild cognitive impairment has linked with poor conditions of physical health in the older population, which increase dependency. The reason behind is lack of awareness and information about this clinical state and symptoms of decline (15). A Japanese study determines the prevalence and related clinical factors associated with cognitive dysfunction in patient with diabetes. Study results revealed that one third of elderly type II diabetes patients had impaired cognitive function and cognition decline was significantly correlated with insulin treatment, age and education level and poor control of diabetes.

\section{Impact of Neurological Changes Associated with Age and Race on Cognitive Dysfunction and Memory Loss}

A cross-sectional study from Denmark reported that the Metabolic Syndrome, Insulin resistance and metabolic profile affect the brain functioning which may lead to changes in neuronal receptors. Data of elder population showed epidemiological risk factor in Denmark, results found that disturbed fasting plasma glucose in elderly patients was associated with $44 \%$ larger probability of developing cognitive dysfunction (16). The risk of both DM and cognitive impairment increases with age, nearly $27 \%$ of adults above 65 years were reported with DM in California (17). A study conducted in University of Maryland U.S examined cognitive dysfunction and functional disability in Adults Aged 75 and older and other risk factors related to it. Study concluded that functional disability and cognitive impairment are associated with increase chances of dependency, their daily activities and reduced quality of life for older adults. Functional decline may be a risk factor for institutionalization, and greater risk for mortality. Health care providers and care takers should be aware of these associations and as well as of preventive care in the elderly patients. Timely detection and preventive action may 
result in delay of functional disability ,memory loss and cognitive dysfunction among diabetes patients (18).

Effect of co-morbid disease or chronic health condition on functional and cognitive decline have been observed in different ethnic groups. A study from USA reported that race can affect the rate of cognitive decline and diabetes. The study showed that incidence of DM in his panic is 10 to 20 years earlier than in non-Hispanic whites. Awareness and knowledge of health and disease related complications that increase the chances for cognitive decline or functional disability in various ethnic groups can delay the risk of dependency in diabetes patients (18).

\section{Effect of Poor Glycemic Control on Cognitive impairment and Memory Loss}

A study conducted in United States as prospective cohort study reported that all existing and new cases of diabetes mellitus (DM) reported with poor glucose control have increased risk of cognitive decline. Several studies were conducted in the University of California reported that poor glucose control among well-functioning adults associated with worse cognitive function and greater decline .The risk of both DM and cognitive impairment increases with unhealthy dietary habits and poor health status (17).A comparative study from Netherland indicated that adults who develop type II diabetes later in life also have cognitive decrements compared with adults without type II diabetes. They were reported with poor glycaemic control, uncontrolled diabetes effect psychomotor efficiency and brain functioning which ultimately effect learning and memory skills (19). A study from United Kingdom indicated that in older age a combination brain changes can lead to cognitive decline and memory loss in diabetes patients. Poor glycaemic control can cause dysfunction in beta cell functioning and changes in neuronal receptors. Insulin resistance may lead to persistent high blood sugar and can cause glucose toxicity which have profound implications for brain cells. This glycemic imbalance in diabetes patients ultimately lead to decline in cognition (20). Another cross-sectional analysis in United States indicated evidence of uncontrolled diabetes to increased estimated cognitive decline. However, vocabulary score which is often used to estimate peak prior cognitive ability was associated with high blood sugar levels in later life (21).

\section{Effect of Hypoglycaemia on Cognitive Impairment and Memory Loss}

Animal studies evaluated that neuronal receptors are responsible for learning and memory, which can get effected because of severe low blood sugar levels in patients with diabetes. These repeated episodes results because of mismanagement in treatment or poor diet. Persistent hypoglycaemia in adults may impair cognition through damage to their neuronal receptors in hippocampus, and cause memory loss (22).The aetiology and pathophysiology of cognitive impairment and memory loss in people with type II diabetes mellitus was probably unknown. A study designed in London suggested that the brains of patient with diabetes mellitus might 
be vulnerable to the effects of repeated episodes of severe hypoglycaemia. Study concluded that cognitive function should now be included in screening and therapeutic interventions in patients with type II diabetes mellitus (23). Looking into effects caused by hypoglycaemia another study assessed relationship of recurrent hypoglycaemia and cognitive impairment in people with diabetes. The study result shows that gradual development of cognitive impairment in Type II diabetes is likely to be complex. People with Type II diabetes who have timely reported about hypoglycaemia showed improved results in their cognitive ability. Hence a detailed literature review proved this association, and suggested that the association may be attributable to an effect of hypoglycaemia on age related cognitive decline (22).

A study conducted in Sweden identified factors that are risk factor for cognitive impairment in diabetes. Study reported that long duration, early age onset of diabetes and presence of peripheral neuropathy had the strongest correlation to cognitive impairment. Diabetes at young age, insulin resistance and poor control for the long time are some other factors (24).

Psychomotor speed, verbal ability and visual perception were those functions, which showed the strongest dependency on any of the disease variables. In contrast to the nerve conduction defects, the cognitive impairment correlate to the presence of long term diabetes, indicating that the pathogenesis of cognitive impairment and peripheral neuropathy is different. Uncontrolled diabetes cause psychomotor changes which effect the executive functions. However, study concluded that the clinical relationships of cognitive impairment and memory loss differ from peripheral neuropathy. These complications have different pathogenesis (24).A study found that both diagnosed and uncontrolled as well as undiagnosed type II diabetes were associated with poor cognitive performance. Slower processing speed in individuals was observed which proceed to psychomotor dysfunctioning and cognitive decline (22)

\section{Impact of Insulin Resistance on Memory Loss and Cognitive Decline}

A study conducted in Netherland provided a comprehensive review of cognitive impairment in patients with type II diabetes mellitus. Study results indicated that DM effect the central nervous system and brain functioning, resulting in impairments of cognitive performance. In young DM I patients cognitive impairment is indicated by slowing of mental speed and a reduced mental flexibility. On the other hand in case of DM II modest impairments in speed of information processing, memory and attention have been noted. Study results concluded that controlled type I patients with duration of 1-30 years of diabetes have better cognitive profile and better MRI ratings. They were under observation, provided with health education and assessed for cognitive complications. Vascular risk factors or co-morbidity such as hypertension, insulin resistance and atherosclerosis are more common in DM II than in DM I, 
that is why type II patients are more prone toward complications including mild cognitive impairment (25).

\section{Relationship of Treatment Regimens and Cognitive Impairment}

Managing diabetes may reduce many risk factors, which may lead to any complication. Patients experience significant difficulty in managing their disease usually reported with mild cognitive impairment and memory loss. They may also have difficulty in treating acute conditions associated with diabetes treatment such as episodes of low sugar levels. A study conducted in Boston U.S reported the relationship between poor glycaemic control and cognitive impairment in adults with metabolic syndrome .This study highlighted the importance of self-management of disease, behaviours (blood glucose testing, meal planning) and barriers in diabetes treatment and medication compliance of diabetes treatment regimens. Study evaluated that such patients are unlikely to self-report any difficulty in diabetes management or self-monitoring of their blood glucose levels as well as cognitive decline. Noncompliance to medication and treatment result poor control of diabetes, which may contribute in cognitive decline. Many healthcare providers may be unaware that their patients have cognitive impairment because of lack of any screening procedure (26).

\section{Relationship of Neurodegenerative Mechanisms with Cognitive Impairment and Memory} Loss

Growing age with prolong period of uncontrolled diabetes represents physiologic changes as well as neuronal changes, which results in neurophysiologic complications in diabetes. Diabetes along number of neurodegenerative mechanisms, cognitive dysfunction and memory loss is a serious problem. Neuron functioning get effected because of hypoglycaemia as well as hyperglycaemia. Persistent elevated blood sugar levels may also be directly toxic to the neuron, leading to its degeneration, as well as changes in brain cells. Hyper insulinemia is a state with diabetes which may contribute in independent pathway of neurodegeneration. However these neuropathology markers of disease leading to memory loss (9).

A Cross-sectional study was conducted in United Kingdom where potential mechanisms were examined by which uncontrolled diabetes cause cognitive decline. Age sensitive neuropsychological tests were performed, results reported neuronal cell damage, impaired nutrient delivery to the brain, increased accumulation of neurotoxin are the causative factors. Damage to neuronal receptors with poor glycaemic control can lead to the neuronal defect (22). An American Comparative study determined Relationship between metabolic syndrome and cognition in patients reported with memory loss. Patients showed significantly worse cognitive functions including processing speed, attention, reasoning, problem solving and vigilance as compared to patients without the diabetes. Study result showed that greater BMI, poor diabetes 
control and higher levels of triglycerides were significantly associated with worse levels of attention and memory. Results reported poorer performance on visual spatial tasks, processing speed and memory in the group of patients with diabetes, while few patients in study showed impaired processing speed, they did not show differences in visual spatial performance which shows the mild cognitive impairment with prolong uncontrolled diabetes (27).

An Australian study assessed relationship between glycaemic control and cognitive function in patients with diabetes in a hospital. Results of Neuropsychological tests shown deficits in various aspects of cognitive function in both young and elderly populations with diabetes. Respective studies have found that higher glycated haemoglobin values were associated with moderate decline in motor speed and psychomotor efficiency. Also found that diabetic individuals have abnormalities in cognitive functions, including a number of complex abilities such as problem solving, planning, organization, insight, reasoning and attention (28).A four years observational study in Netherlands examined the relationship between micro vascular complications, development of structural brain abnormalities and cognitive decline in patients with diabetes mellitus. The study reported a gradual decline in information processing speed, attention and executive functioning; same trend in same direction was examined for memory. Study concluded that these changes usually occurs after prolong time period (29).

\section{Overview of Cognitive Decline among Diabetes Patients in Developing Countries}

Age-related cognitive impairment associated to diabetes with negative outcomes including functional disability, decrease in quality of life, and early mortality have been reported in Singapore (30).A systematic review from China based on age related cognitive decline provided an estimation of cognitive decline which shows that 35.6 million people worldwide were living with dementia and mild cognitive impairment in 2010, and this number is projected nearly to double every 20 years. According to the International Diabetes Federation, diabetes affects at least 382 million people worldwide, and that number is expected to reach 592 million by the year 2035 (31). Diabetes management, treatment and self-care in persons with type II diabetes have consequences for better or worse health status. Diabetes self-management plays an important role in prevalence of type II diabetes and complications including cognitive performances in high-risk individuals. Study concluded that future treatment protocols and preventive strategies should be developed with the screening of cognitive status of patients with diabetes (9).

An Indian cross sectional study conducted with aim to examine the prevalence and risk factors of cognitive dysfunction in patients with type II diabetes mellitus and to assess its influence on medication adherence showed that $14 \%$ of the selected sample had cognitive dysfunction but no significant association was found between cognitive dysfunction and treatment adherence. 
Study results concluded that one out of seven of our patients with type II diabetes have cognitive dysfunction, which is strongly associated with low level of education and does not affect treatment adherence and glycaemic control (32).

Several studies results reported that end organ damage in diabetes is a serious problem for health care providers, patient and families. Changes in cerebral structure and function are also complication of diabetes, which are related to end organ damage due to uncontrolled diabetes. Macro vascular disease, hypoglycaemia, insulin resistance, poor glycaemic control and changes in brain structure like amyloidal lesions may act as risk factor in some patients. However uncontrolled and mismanaged cases has greater chances to induce cognitive decline (7).

Diabetes itself might not be a risk factor but mismanagement and poor control of disease may lead to the problem .National Health and Nutrition Examination Survey (NHANES) database in China for the years 1999 to 2002 conducted a study and reported the relationship between diabetes and cognitive decline. Study results concluded that high blood glucose, elevated blood pressure, obesity, and high cholesterol collectively lead to complications including decline in cognitive ability (3). Health and Retirement Study (HRS) Mail Survey on Diabetes in 2003 and the 2004 wave of the HRS concluded that Glycosylated haemoglobin (HbA1c) level, uncontrolled diabetes and hypoglycaemia are the risk factors along age which may lead to cognitive complications (33). Diabetes mellitus was reported to be only associated with a more rapid rate of decline in perceptual speed (34).

\section{Overview of Cognitive Decline among Diabetes Patients in Pakistan}

Diabetes Mellitus has been reported as one of the greatest endemic public health challenge in Pakistan. It is not only a risk factors for CVS and renal failure patients, but is also accountable for other sever $\&$ dangerous diseases of brain like dementia, memory loss $\&$ abnormal cognitive perception. A study conducted in Karachi, Pakistan reported memory loss is the major risk factors found after diabetes in people almost with all age groups accept younger.

\section{Interventions for Improving Cognition in Diabetes Patients}

An interventional study was conducted in Netherland to examine Cognitive function in patients with diabetes mellitus and to provide guidance for daily care. The results concluded that these diabetes-associated cognitive decrements can effects daily routine activity and diabetes selfmanagement. Cognitive decline in diabetics and structural brain changes are detectable with brain MRI, but usually show little progression over time. Although cognitive decrements do not generally represent a pre-dementia stage that is mild impairment in patients less than age 60years of age, which shows that age dependent cognitive impairment can be seen in diabetes patients (1). 
Literature review shows that acknowledgment of diabetes associated cognitive impairment and memory loss can help to improve symptoms of impairment and management can delay the consequences. Screening procedures for cognitive impairment in people with diabetes should be established, which can help to identify those who are at increased risk of accelerated cognitive decline at an early stage. This can also help to develop effective treatments regimen. Self-reported severe hypoglycaemia was associated with reduced late-life general cognitive ability among diabetics, independent of diabetes duration and vascular disease. Severe hypoglycaemia episodes and subsequent cognitive decline will have important implications for the management of older people with Type II diabetes. Where highly intensive glycaemic control, using anti-diabetic agents that promote hypoglycaemia, may be contraindicated to avoid the side effects (1).

An interventional study investigated that the duration of diabetes, glycated haemoglobin levels and glycaemic fluctuations were associated with cognitive decline and dementia. Furthermore, cognitive impairment and memory loss were associated with poorer diabetes management, poor control and recurrent low sugar level. So this is highly concerned with health care providers. Data from literature review provides evidence of the association between diabetes, cognitive decline and memory loss. Diabtes patients with dementia could benefit from structured educational preventive strategies, which involve empowerment and education programmes and lifestyle modifications. These preventive measurements can delay the risk of cognitive decline (20). Controlled participants with diabetes, interventions and regular follow up can improve management of diabetes (10).

An interventional study conducted in Sweden for diet, exercise, cognitive training, and vascular risk monitoring to prevent cognitive decline in at-risk elderly people. The duration of study was two years. Participants aged 60-77 years were enrolled in randomized controlled trial. Findings from this large, long-term, randomised controlled trial suggest that a multi domain intervention improve or maintain cognitive functioning in at-risk diabetes patients (35). University of California provided Promising Strategies for the Prevention of memory loss and cognitive decline in diabetes. Lifestyle modifications and biological factors were considered crucial in preventive measurements. Intervention combine a number of factors, such as healthy nutrition along with cognitive, social, and physical activity, healthy life style, glycaemic control and medicine compliance. In the result, cognitive impairment and memory loss could be delayed or even prevented by these preventive strategies or interventions. As a result, patient can improve their overall health, especially their cardiovascular health, and would be able to enjoy a more cognitively and socially engaging life (36). 


\section{CONCLUSION}

The present review concluded that for successful management of diabetes, it is important to identify the degree of cognitive impairment and memory loss in patients. Regular screening for cognitive impairment among diabetes patients must be conducted in order to provide optimal patient care. Educational programs should be designed to improve knowledge of disease among diabetics. In future, qualitative studies must be designed to explore the reason behind cognitive decline among diabetes patients underlying treatment. Longitudinal studies should be conducted to explore the factors associated with diabetes effecting cognition and memory loss. Moreover, the impact of and poor glycaemic control and medication non adherence on psychomotor functions in diabetes patients must also be explored.

\section{REFERENCES}

1. Koekkoek PS, Kappelle LJ, van den Berg E, Rutten GE, Biessels GJ. Cognitive function in patients with diabetes mellitus: guidance for daily care. The Lancet Neurology. 2015;14(3):329-40.

2. Medalia A, Revheim N. Dealing with cognitive dysfunction associated with psychiatric disabilities: A handbook for families and friends of individuals with psychiatric disorders: New York State Office of Mental Health; 2002.

3. Tsai C-K, Kao T-W, Lee J-T, Wu C-J, Hueng D-Y, Liang C-S, et al. Increased risk of cognitive impairment in patients with components of metabolic syndrome. Medicine. 2016;95(36).

4. Shaik MA, Chan QL, Xu J, Xu X, Hui RJY, Chong SST, et al. Risk factors of cognitive impairment and brief cognitive tests to predict cognitive performance determined by a formal neuropsychological evaluation of primary health care patients. Journal of the American Medical Directors Association. 2016;17(4):343-7.

5. Simó R, Ciudin A, Simó-Servat O, Hernández C. Cognitive impairment and dementia: a new emerging complication of type 2 diabetes-The diabetologist's perspective. Acta diabetologica. 2017:1-8.

6. Formiga F, Ferrer A, Padrós G, Corbella X, Cos L, Sinclair AJ, et al. Diabetes mellitus as a risk factor for functional and cognitive decline in very old people: The Octabaix study. Journal of the American Medical Directors Association. 2014;15(12):924-8.

7. Wennberg AM, Hagen CE, Gottesman RF, Zipunnikov V, Kaufmann CN, Albert MS, et al. Longitudinal association between diabetes and cognitive decline: The National Health and Aging Trends Study. Archives of gerontology and geriatrics. 2017;72:3944. 
8. Kim B, Feldman EL. Insulin resistance as a key link for the increased risk of cognitive impairment in the metabolic syndrome. Experimental \& molecular medicine. 2015;47(3):e149.

9. Saczynski JS, Jónsdóttir MK, Garcia ME, Jonsson PV, Peila R, Eiriksdottir G, et al. Cognitive impairment: an increasingly important complication of type 2 diabetes: the age, gene/environment susceptibility-Reykjavik study. American journal of epidemiology. 2008;168(10):1132-9.

10. Feinkohl I, Aung PP, Keller M, Robertson CM, Morling JR, McLachlan S, et al. Severe hypoglycemia and cognitive decline in older people with type 2 diabetes: the Edinburgh Type 2 Diabetes Study. Diabetes care. 2014;37(2):507-15.

11. Paddick S-M, Gray WK, McGuire J, Richardson J, Dotchin C, Walker RW. Cognitive screening tools for identification of dementia in illiterate and low-educated older adults, a systematic review and meta-analysis. International Psychogeriatrics. 2017;29(6):897929.

12. Hunt HA, Van Kampen S, Takwoingi Y, Llewellyn DJ, Pearson M, Hyde CJ. The comparative diagnostic accuracy of the Mini Mental State Examination (MMSE) and the General Practitioner assessment of Cognition (GPCOG) for identifying dementia in primary care: a systematic review protocol. Diagnostic and Prognostic Research. 2017;1(1):14.

13. Moattari M, Jamalnia S, Mansoori P, Mani A, Dabbaghmanesh MH, Sayadi M. The prevalence of Cognitive Impairment in Patients with Type II Diabetes and its Relationship with Quality of Life, Self-Management Profiles, and HbA1c. Iranian Journal of Diabetes \& Obesity (IJDO). 2016;8(1).

14. Gorska-Ciebiada M, Saryusz-Wolska M, Ciebiada M, Loba J. Mild cognitive impairment and depressive symptoms in elderly patients with diabetes: prevalence, risk factors, and comorbidity. Journal of diabetes research. 2014;2014.

15. Frisoni GB, Fratiglioni L, Fastbom J, Guo Z, Viitanen M, Winblad B. Mild cognitive impairment in the population and physical health: data on 1,435 individuals aged 75 to 95. The Journals of Gerontology Series A: Biological Sciences and Medical Sciences. 2000;55(6):M322-M8.

16. Neergaard JS, Dragsbæk K, Christiansen C, Nielsen HB, Brix S, Karsdal MA, et al. Metabolic Syndrome, Insulin Resistance and Cognitive Dysfunction: Does your metabolic profile affect your brain? Diabetes. 2017:db161444. 
17. Yaffe K, Falvey C, Hamilton N, Schwartz AV, Simonsick EM, Satterfield S, et al. Diabetes, glucose control, and 9-year cognitive decline among older adults without dementia. Archives of neurology. 2012;69(9):1170-5.

18. Black SA, Rush RD. Cognitive and functional decline in adults aged 75 and older. Journal of the American Geriatrics Society. 2002;50(12):1978-86.

19. Biessels GJ, Deary IJ, Ryan CM. Cognition and diabetes: a lifespan perspective. The Lancet Neurology. 2008;7(2):184-90.

20. Ojo O, Brooke J. Evaluating the association between diabetes, cognitive decline and dementia. International journal of environmental research and public health. 2015;12(7):8281-94.

21. Marioni RE, Deary IJ, Strachan MW, Lowe GD, Rumley A, Murray GD, et al. Blood rheology and cognition in the Edinburgh Type 2 Diabetes Study. Age and ageing. 2010;39(3):354-9.

22. Aung P, Strachan M, Frier B, Butcher I, Deary I, Price J. Severe hypoglycaemia and late-life cognitive ability in older people with Type 2 diabetes: the Edinburgh Type 2 Diabetes Study. Diabetic Medicine. 2012;29(3):328-36.

23. Strachan MW, Reynolds RM, Marioni RE, Price JF. Cognitive function, dementia and type 2 diabetes mellitus in the elderly. Nature Reviews Endocrinology. 2011;7(2):10814.

24. Brismar T, Maurex L, Cooray G, Juntti-Berggren L, Lindström P, Ekberg K, et al. Predictors of cognitive impairment in type 1 diabetes. Psychoneuroendocrinology. 2007;32(8):1041-51.

25. Brands AM, Biessels GJ, Kappelle LJ, de Haan EH, de Valk HW, Algra A, et al. Cognitive functioning and brain MRI in patients with type 1 and type 2 diabetes mellitus: a comparative study. Dementia and geriatric cognitive disorders. 2007;23(5):343-50.

26. Munshi M, Grande L, Hayes M, Ayres D, Suhl E, Capelson R, et al. Cognitive dysfunction is associated with poor diabetes control in older adults. Diabetes care. 2006;29(8):1794-9.

27. Lindenmayer JP, Khan A, Kaushik S, Thanju A, Praveen R, Hoffman L, et al. Relationship between metabolic syndrome and cognition in patients with schizophrenia. Schizophrenia Research. 2012;142(1):171-6.

28. Mahakaeo S, Zeimer H, Woodward M. Relationship between glycemic control and cognitive function in patients with type 2 diabetes in a hospital aged care unit. European Geriatric Medicine. 2011;2(4):204-7. 
29. Van den Berg E, Reijmer Y, De Bresser J, Kessels R, Kappelle L, Biessels G, et al. A 4 year follow-up study of cognitive functioning in patients with type 2 diabetes mellitus. Diabetologia. 2010;53(1):58-65.

30. Ong SY, Cheung CY, Lamoureux E, Saw S-M, Venketasubramanian N, Chen CP, et al. Age-related Eye Diseases and Cognitive Impairment: The Singapore Malay Eye Study. Investigative Ophthalmology \& Visual Science. 2011;52(14):4233-.

31. Li J, Cesari M, Liu F, Dong B, Vellas B. Effects of diabetes mellitus on cognitive decline in patients with Alzheimer disease: a systematic review. Canadian journal of diabetes. 2017;41(1):114-9.

32. Abba ZI, Mboue-Djieka Y, Mapoure YN, Nkouonlack C, Luma HN, Choukem S-P. Prevalence and risk factors of cognitive dysfunction in patients with type 2 diabetes mellitus receiving care in a reference hospital in Cameroon: a cross-sectional study. International Journal of Diabetes in Developing Countries. 2017:1-7.

33. Okura T, Heisler M, Langa KM. Association between cognitive function and social support with glycemic control in adults with diabetes mellitus. Journal of the American Geriatrics Society. 2009;57(10):1816-24.

34. Arvanitakis Z, Wilson RS, Bienias JL, Evans DA, Bennett DA. Diabetes mellitus and risk of Alzheimer disease and decline in cognitive function. Archives of neurology. 2004;61(5):661-6.

35. Ngandu T, Lehtisalo J, Solomon A, Levälahti E, Ahtiluoto S, Antikainen R, et al. A 2 year multidomain intervention of diet, exercise, cognitive training, and vascular risk monitoring versus control to prevent cognitive decline in at-risk elderly people (FINGER): a randomised controlled trial. The Lancet. 2015;385(9984):2255-63.

36. Middleton LE, Yaffe K. Promising strategies for the prevention of dementia. Archives of neurology. 2009;66(10):1210-5.

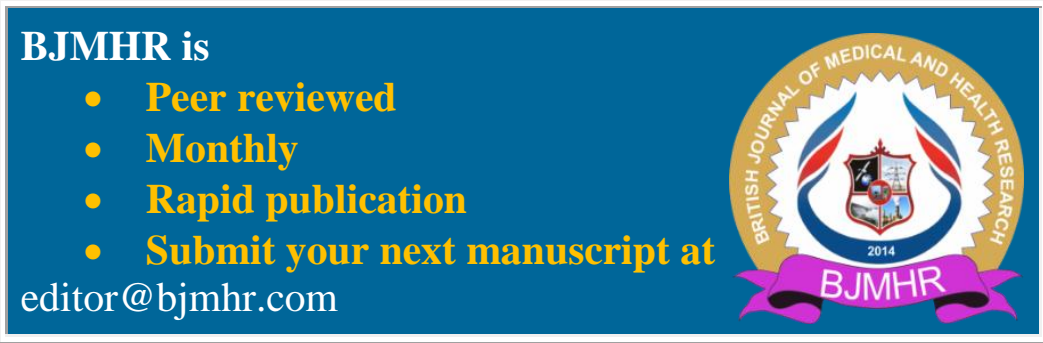

